\title{
Adaptive Wavelet Precise Integration Method for Nonlinear Black-Scholes Model Based on Variational Iteration Method
}

\author{
Huahong Yan \\ School of Accounting, Capital University of Economics and Business, 121 Zhangjialukou, Huaxiang Fengtai District, \\ Beijing 100070, China \\ Correspondence should be addressed to Huahong Yan; yanhuahong@263.net
}

Received 31 December 2012; Revised 14 February 2013; Accepted 17 February 2013

Academic Editor: Lan Xu

Copyright (C) 2013 Huahong Yan. This is an open access article distributed under the Creative Commons Attribution License, which permits unrestricted use, distribution, and reproduction in any medium, provided the original work is properly cited.

\begin{abstract}
An adaptive wavelet precise integration method (WPIM) based on the variational iteration method (VIM) for Black-Scholes model is proposed. Black-Scholes model is a very useful tool on pricing options. First, an adaptive wavelet interpolation operator is constructed which can transform the nonlinear partial differential equations into a matrix ordinary differential equations. Next, VIM is developed to solve the nonlinear matrix differential equation, which is a new asymptotic analytical method for the nonlinear differential equations. Third, an adaptive precise integration method (PIM) for the system of ordinary differential equations is constructed, with which the almost exact numerical solution can be obtained. At last, the famous Black-Scholes model is taken as an example to test this new method. The numerical result shows the method's higher numerical stability and precision.
\end{abstract}

\section{Introduction}

The Black-Scholes equation is a mathematical model of a financial market containing certain derivative investment instruments (definition). The idea behind the Black-Scholes model is that the price of an option is determined implicitly by the price of the underlying stock. The Black-Scholes model is a mathematical model based on the notion that prices of stock follow a stochastic process. It is widely employed as a useful approximation, but proper application requires understanding its limitations. Therefore, many nonlinear BlackScholes equations are proposed in recent years $[1,2]$. But it is very difficult to obtain the exact analytical solutions of the nonlinear Black-Scholes models. There are some numerical algorithms that have been proposed based on the difference method to solve those nonlinear problems, but the precision depends on the time step and the discretization in definition domain $[3,4]$.

Variational iteration method [5-9] proposed by $\mathrm{He}$ is a new analytical method to solve nonlinear differential equations, which has been rapidly developed to solve various nonlinear problems of science and engineering as its flexibility and ability to solve nonlinear equations accurately and conveniently [10]. The typical application includes solving freeconvective boundary-layer equation [11], $q$-difference equations $[12,13]$, and Burgers' flow with fractional derivatives $[14,15]$. Comparing with the traditional numerical methods, VIM needs no discretization, linearization, transformation, or perturbation. The wavelet precise integration method (WPIM) is a simple and effective method for linear partial differential equations proposed by Mei [16-20]. For linear steady structural dynamic systems, its numerical results at the integration points are almost equal to that of the exact solution in machine accuracy. But in solving the nonlinear partial differentials, the time step has to be limited to a small value in WPIM for high accuracy.

The main purpose of this paper is to construct a modified VIM for nonlinear Black-Scholes model with combining the VIM with WPIM. According to WPIM, the nonlinear differential equation should be transformed to a system of ordinary differential equations with the multiscales wavelet interpolation operator, and then the nonlinear PDEs become a system of nonlinear ordinary differential equations. So solving the matrix differential equation (MDE) is the key in solving 
nonlinear PDEs with WPIM. In fact, the matrix differential equation (MDE) is a crucial mathematical foundation of the system engineering and the control theory. But most matrix differential equations do not have precise analytical solutions except linear time-invariant system. In this paper, a coupling technique of He's VIM and WPIM is developed to establish an approximate analytical solution of the matrix differential equations. In contrast to the traditional finite difference approximation, the numerical result obtained with PIM for a set of simultaneous linear time-invariant ODEs approaches the computer precision and is also free from the stiff problem.

\section{Fundamental Theory of Coupling Technique of VIM and WPIM}

2.1. VIM for Matrix Differential Equation. Consider the nonlinear matrix differential equations as follows:

$$
L(\dot{\mathbf{V}}, \mathbf{V}, t)+N(\dot{\mathbf{V}}, \mathbf{V}, t)=\mathbf{G}(t),
$$

where $L$ is a linear operator, $N$ is a nonlinear operator, $\mathbf{G}(t)$ is an inhomogeneous term, $\mathbf{V}$ is an $n$-dimensional unknown vector, and dot stands for the differential with respect to time variable $t$. For convenience, (1) can be rewritten as

$$
\dot{\mathbf{V}}-\mathbf{H V}-\mathbf{F}(\dot{\mathbf{V}}, \mathbf{V}, t)=0,
$$

where $\mathbf{H}$ is a given $n \times n$ constant matrix, and $\mathbf{F}(\dot{\mathbf{V}}, \mathbf{V}, t)$ is a $n$-dimensional nonlinear external force vector.

According to VIM, we can write down a correction functional as follows:

$$
\begin{aligned}
& \mathbf{V}_{n+1}(t) \\
& \quad=\mathbf{V}_{n}(t)+\int_{0}^{t} \lambda\left[\dot{\mathbf{V}}_{n}(\tau)-\mathbf{H} \mathbf{V}_{n}(\tau)-\mathbf{F}\left(\dot{\tilde{\mathbf{V}}}_{n}, \tilde{\mathbf{V}}_{n}, \tau\right)\right\rfloor d \tau,
\end{aligned}
$$

where $\lambda$ is a general Lagrange vector multiplier $[4,5,8]$ which can be identified optimally via the variational theory. The subscript $n$ denotes the $n$th approximation, and $\widetilde{\mathbf{V}}_{n}$ is considered as a restricted variation [13-15]; that is, $\delta \widetilde{\mathbf{V}}_{n}=0$.

Using VIM, the stationary conditions of (3) can be obtained as follows:

$$
\begin{gathered}
\lambda^{\prime}+\lambda \mathbf{H}=0, \\
1+\left.\lambda(\tau)\right|_{\tau=t}=0 .
\end{gathered}
$$

The Lagrange vector multiplier can therefore be readily identified as follows:

$$
\lambda(\tau)=-e^{\mathbf{H}(t-\tau)}
$$

As a result, we obtain the following iteration formula:

$$
\begin{array}{r}
\mathbf{V}_{n+1}(t)=\mathbf{V}_{n}(t)-\int_{0}^{t} e^{\mathbf{H}(t-\tau)} \mid \dot{\mathbf{V}}_{n}(\tau) \mathbf{H} \mathbf{V}_{n}(\tau)- \\
\left.-\mathbf{F}\left(\dot{\tilde{\mathbf{V}}}_{n}, \tilde{\mathbf{V}}_{n}, \tau\right)\right\rfloor d \tau .
\end{array}
$$

According to VIM, we can start with an arbitrary initial approximation that satisfies the initial condition. So we take the exact analytical solution of $\mathbf{V}-\mathbf{H V}=0$ as the initial approximation; that is,

$$
\mathbf{V}_{0}(t)=e^{\mathbf{H} t} \mathbf{A},
$$

where $\mathbf{A}$ is the given initial value vector.

Substituting (7) into (6) and after simplification, we have

$$
\mathbf{V}_{n+1}(t)=\mathbf{V}_{n}(t)+\int_{0}^{t} e^{\mathbf{H}(t-\tau)} \mathbf{F}\left(\dot{\tilde{\mathbf{V}}}_{n}, \tilde{\mathbf{V}}_{n}, \tau\right) d \tau
$$

According to the theory of matrices, the analytical expression of the external force $\mathbf{F}\left(\dot{\widetilde{\mathbf{V}}}_{n}, \widetilde{\mathbf{V}}_{n}, \tau\right)$ is required now, but it is not always available, except $\mathbf{F}\left(\dot{\tilde{\mathbf{V}}}_{n}, \widetilde{\mathbf{V}}_{n}, \tau\right)$ is a constant vector f; that is,

$$
\mathbf{F}\left(\dot{\tilde{\mathbf{V}}}_{n}, \tilde{\mathbf{V}}_{n}, \tau\right)=\mathbf{f}
$$

the integration term of (8) is

$$
\int_{0}^{t} e^{\mathbf{H}(t-\tau)} \mathbf{f} d \tau=\left(e^{\mathbf{H} t}-\mathbf{I}\right) \mathbf{H}^{-1} \mathbf{f}
$$

where the exponential matrix $e^{\mathbf{H} t}$ can be calculated accurately in PIM and $\mathbf{I}$ is a unit matrix.

Substituting (10) into (8), we obtain the variational iteration formula of the matrix differential equation:

$$
\mathbf{V}_{n+1}(t)=\mathbf{V}_{n}(t)+\left(e^{\mathbf{H} t}-\mathbf{I}\right) \mathbf{H}^{-1} \mathbf{f}
$$

2.2. Coupling Technique of VIM and WPIM for Nonlinear Partial Differential Equation. In most cases, the second-order nonlinear PDEs about the unknown function $u(t, x)$ can be expressed as follows:

$$
F\left(u, u_{t}, u_{x}, u_{t, x}, u_{x x}\right)=0
$$

In order to transform the previous nonlinear PDEs into the matrix ODEs form as (1), an adaptive multilevels wavelet interpolation operator should be constructed firstly.

In this section, we take the quasi-Shannon wavelet function as the basis function to approximate the solution function of the nonlinear PDEs. The quasi-Shannon function is defined as follows:

$$
\delta_{\Delta \sigma}(x)=\frac{\sin (\pi x / \Delta)}{\pi x / \Delta} \exp \left(-\frac{x^{2}}{2 \sigma^{2}}\right),
$$

where $\Delta$ is the discrete step and $\sigma=r \Delta$ ( $r$ is a constant $)$ is a parameter relative to the size of the window.

To construct the multilevel interpolation wavelet operator, it is necessary to discretize the wavelet function and the solution function $u(x)$ evenly in the definition domain $[a, b]$. Let the amount of the discrete points be $2^{j}+1(j \in Z)$, and then the discrete points can be defined as

$$
x_{j}^{i}=a+\frac{i(b-a)}{2^{j}} .
$$


The corresponding discrete basis function can be rewritten as

$$
\varphi_{j}^{i}(x)=\frac{\sin \left(2^{j} \pi /(b-a)\right)\left(x-x_{i}\right)}{\left(2^{j} \pi /(b-a)\right)\left(x-x_{i}\right)} \exp \left(-\frac{2^{2 j-1}\left(x-x_{i}\right)^{2}}{r^{2}(b-a)^{2}}\right) .
$$

The interpolation operator can be defined as

$$
u_{J}(x)=\sum_{i \in Z_{\Omega}^{J}} I_{i}(x) u_{J}^{i}, \quad Z_{\Omega}^{J}:=0,1,2, \ldots, 2^{J},
$$

where $I_{i}(x)$ is the interpolation function. According to the wavelet transform theory, function $u(x)$ can be expressed approximately as

$$
u_{J}(x)=\sum_{k_{0}=0}^{2^{j_{0}}} u\left(x_{j_{0}}^{k_{0}}\right) \varphi_{j_{0}}^{k_{0}}(x)+\sum_{j=j_{0}}^{J-1} \sum_{k \in Z^{j}} \alpha_{j}^{k} \psi_{j}^{k}(x),
$$

where $Z^{j}:=0,1,2, \ldots, 2^{j}$ and the interpolation wavelet transform coefficient can be denoted as

$$
\begin{aligned}
\alpha_{j}^{k}= & u\left(x_{j+1}^{2 k+1}\right)-\left[\sum_{k_{0}=0}^{2^{j_{0}}} u\left(x_{j_{0}}^{k_{0}}\right) \varphi_{j_{0}}^{k_{0}}\left(x_{j+1}^{2 k+1}\right)\right. \\
& \left.+\sum_{j_{1}=j_{0}}^{j-1} \sum_{k_{1}=0}^{2^{j_{1}-1}} \alpha_{j_{1}}^{k_{1}} \psi_{j_{1}}^{k_{1}}\left(x_{j+1}^{2 k+1}\right)\right] \\
= & \sum_{n=0}^{2^{J}}\left[R_{j+1, J}^{2 k+1, n}-\sum_{k_{0}=0}^{2^{j_{0}}} R_{j_{0}, J}^{k_{0}, n} \varphi_{j_{0}}^{k_{0}}\left(x_{j+1}^{2 k+1}\right)\right] u\left(x_{J}^{n}\right) \\
& -\sum_{n=0}^{2^{J}} \sum_{j_{1}=j_{0}}^{j-1} \sum_{k_{1}=0}^{2^{j_{1}-1}} \alpha_{j_{1}}^{k_{1}} \psi_{j_{1}}^{k_{1}}\left(x_{j+1}^{2 k+1}\right),
\end{aligned}
$$

where $0 \leq j \leq J-1, k \in Z^{j}, n \in Z^{J}$, and $R$ is the restriction operator defined as

$$
R_{l, j}^{i, m}= \begin{cases}1, & x_{l}^{i}=x_{j}^{m} \\ 0, & \text { others. }\end{cases}
$$

Suppose that

$$
\alpha_{j}^{k}=\sum_{n=0}^{2^{J}} C_{j, J}^{k, n} u\left(x_{J}^{n}\right)
$$

Substituting (20) into (18), we can obtain

$$
\begin{aligned}
C_{j, J}^{k, n}= & R_{j+1, J}^{2 k+1, n}-\sum_{k_{0}=0}^{2^{j_{0}}} R_{j_{0}, J}^{k_{0}, n} \varphi_{j_{0}}^{k_{0}}\left(x_{j+1}^{2 k+1}\right) \\
& -\sum_{j_{1}=j_{0}}^{j-1} \sum_{k_{1}=0}^{2^{j_{1}-1}} C_{j_{1}, J}^{k_{1}, n} \psi_{j_{1}, k_{1}}\left(x_{j+1}^{2 k+1}\right) .
\end{aligned}
$$

If $j=j_{0}$, then

$$
C_{j, J}^{k, n}=R_{j+1, J}^{2 k+1, n}-\sum_{k_{0}=0}^{2^{j 0}} R_{j_{0}, J}^{k_{0}, n} \varphi_{j_{0}}^{k_{0}}\left(x_{j+1}^{2 k+1}\right) .
$$

Substituting the restriction operator (19) and the wavelet transform coefficient (20) into (17), the approximate expression of the solution function $u(x)$ can be obtained as

$$
\begin{aligned}
u_{J}(x)=\sum_{i \in Z^{J}}( & \sum_{k_{0}=0}^{2^{j}} R_{j_{0}, J}^{k_{0}, n} \varphi_{j_{0}}^{k_{0}}\left(x_{j+1}^{2 k+1}\right) \\
& \left.+\sum_{j_{1}=j_{0}}^{j-1} \sum_{k_{1}=0}^{2^{j_{1}-1}} C_{j_{1}, J}^{k_{1}, n} \psi_{j_{1}, k_{1}}\left(x_{j+1}^{2 k+1}\right)\right) u\left(x_{J}^{i}\right) .
\end{aligned}
$$

According to the definition of the interpolation operator (16), it is easy to obtain the expression of the interpolation operator

$$
I_{i}(x)=\sum_{k_{0}=0}^{2^{j_{0}}} R_{j_{0}, J}^{k_{0}, i} \varphi_{j_{0}}^{k_{0}}(x)+\sum_{j=j_{0}}^{J-1} \sum_{k \in Z^{j}} C_{j, J}^{k, i} \psi_{j}^{k}(x) .
$$

The corresponding $m$-order derivate of the interpolation operator is

$$
D_{i}^{(m)}(x)=\sum_{k_{0}=0}^{2^{j_{0}}} R_{j_{0}, J}^{k_{0}, i} \varphi_{j_{0}, k_{0}}^{(m)}(x)+\sum_{j=j_{0}}^{J-1} \sum_{k \in Z^{j}} C_{j, J}^{k, i} \psi_{j, k}^{(m)}(x) .
$$

Substituting (24) and (25) into (12), the nonlinear PDEs can be changed into an nonlinear ODEs like (1), and then the corresponding analytical solution can be obtained with (11).

In order to solve (1) accurately, the exponential matrix $T(t)=e^{\mathbf{H} t}$ can be calculated accurately by WPIM as follows:

$$
T(t)=\exp (\mathbf{H} t)=\left[\exp \left(\frac{\mathbf{H} t}{2^{N}}\right)\right]^{2^{N}} .
$$

Let $\Delta t=\tau / 2^{N}$, where $N$ is a positive integer (usually take $N=20$, and then $\Delta t=\tau / 1048576)$. As $\tau$ is a small time step, $\Delta t$ is a much smaller value, then

$$
\begin{aligned}
\exp (\mathbf{H} t) & =I+\mathbf{T}_{\mathbf{a}} \\
& =I+\mathbf{H} t+\frac{(\mathbf{H} t)^{2}\left[I+(\mathbf{H} t) / 3+(\mathbf{H} t)^{2} / 12\right]}{2}
\end{aligned}
$$

which is the Taylor series expansion of $\exp (\mathbf{H} \Delta t)$. In order to calculate the matrix $T$ more accurately, it is necessary to factorize the matrix $T$ as

$$
\mathbf{T}(t)=[\exp (\mathbf{H} t)]^{2^{N}}=\left(I+\mathbf{T}_{\mathrm{a}}\right)^{2^{N-1}}\left(I+\mathbf{T}_{\mathrm{a}}\right)^{2^{N-1}} .
$$

After doing $N$ times of factorization as mentioned above, a more accurate solution of $T$ can be obtained.

The calculation of the exponent matrix $T(i h)$ at different time steps is needed in solving nonlinear equations through iteration based on the precise integration method, and the algorithm of the matrix $T(i h)$ presented here can obtain all the matrices at different time steps for once.

\section{Coupling Technique of VIM and WPIM for the Nonlinear Black-Scholes Model}

In order to test the accuracy of the coupling technique of VIM and WPIM for solving nonlinear PDEs, we will consider 


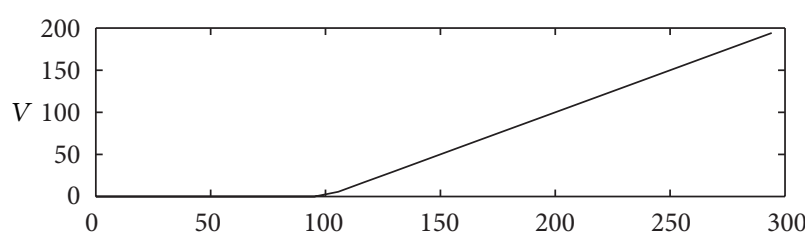

(a)

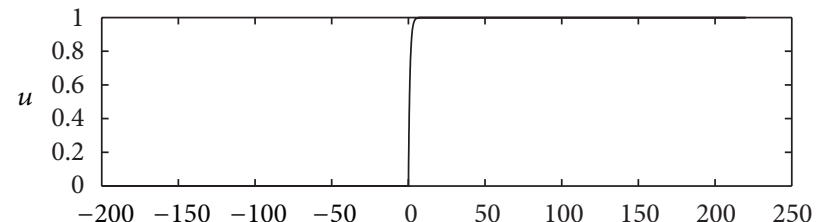

(b)

FIGURE 1: Initial condition of Black-Scholes model.
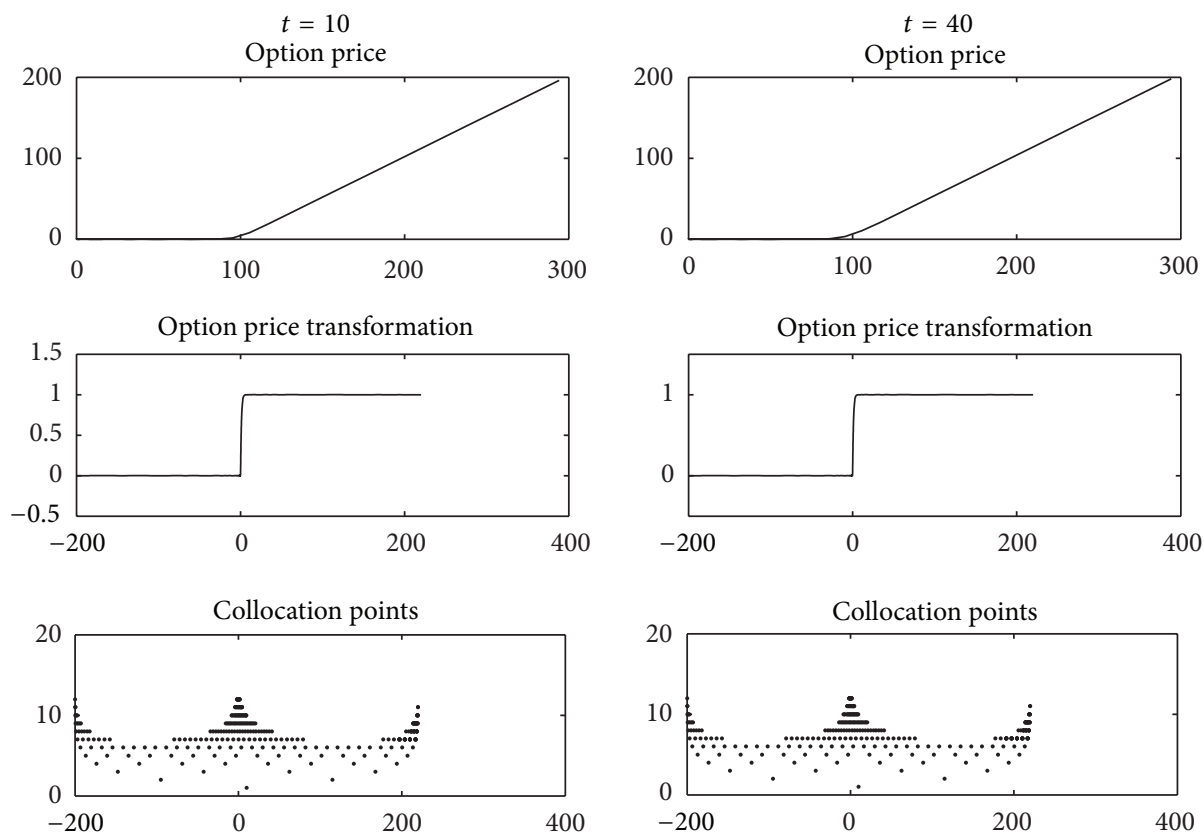

(a)

(b)

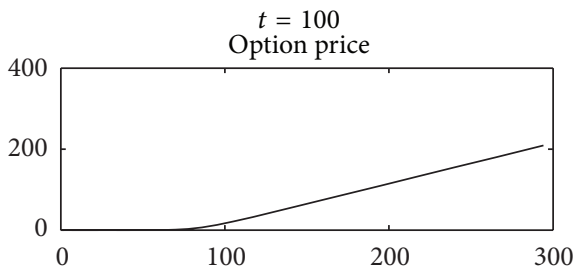

$t=200$
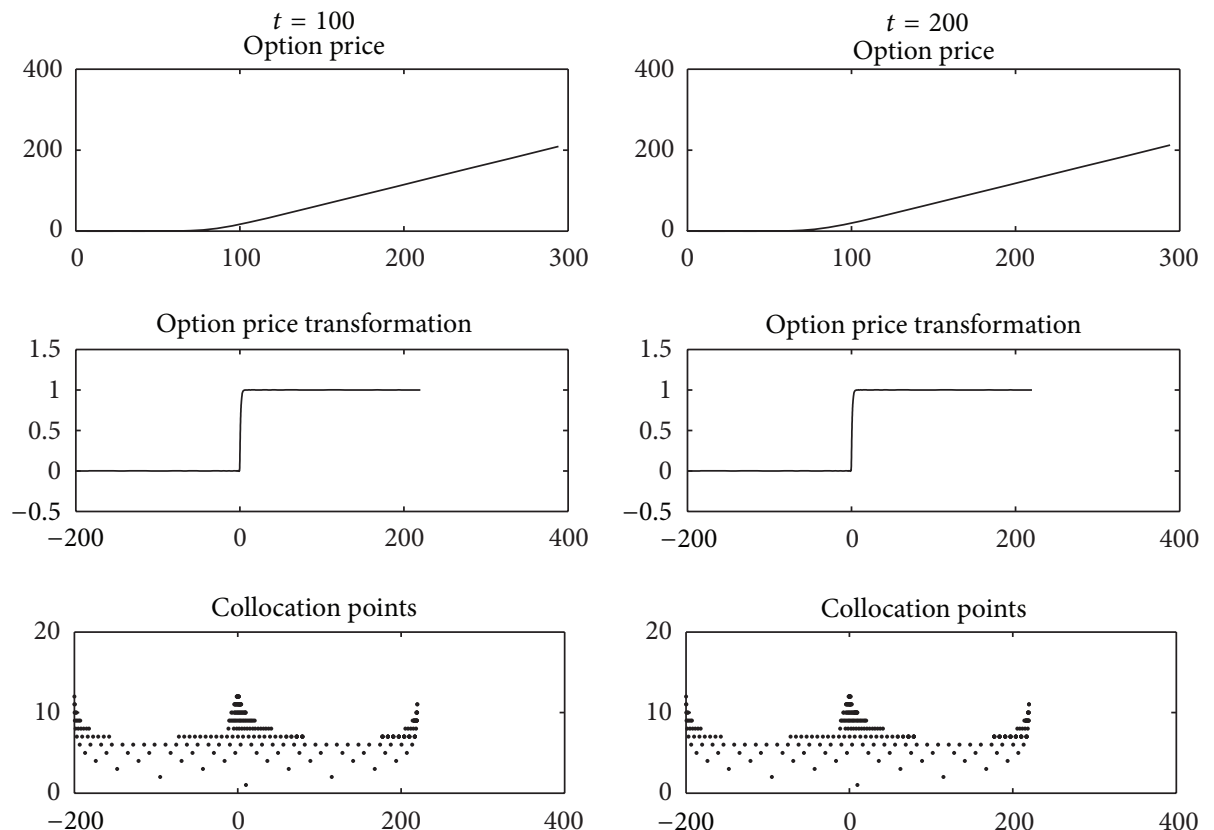

(c)

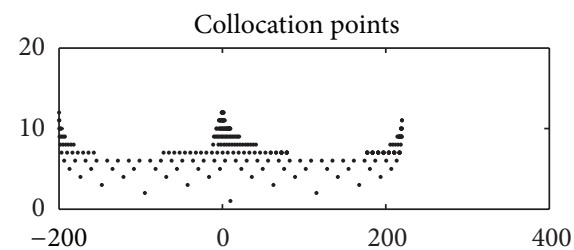

(d)

FIgURE 2: Evolution of the call option price with the parameter $t$. 


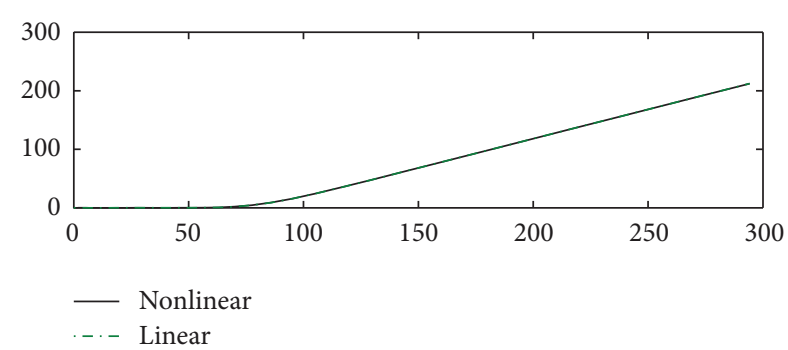

(a)

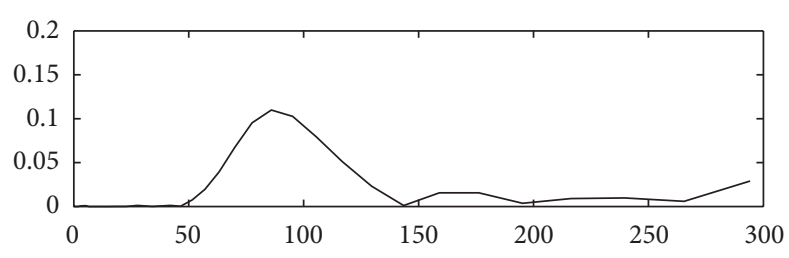

(b)

FIGURE 3: Error of call option price between the linear and nonlinear Black-Scholes models.

the nonlinear Black-Scholes equations which have been increasingly attracting interest over the last two decades, since they provide more accurate values by taking into account more realistic assumptions, such as transaction costs, risks from an unprotected portfolio, large investor's preferences, or illiquid markets, which may have an impact on the stock price, the volatility, the drift, and the option price itself.

Consider the Black-Scholes equation:

$$
\frac{\partial V}{\partial t}=r V-\frac{1}{2} \sigma^{2} S^{2} \frac{\partial^{2} V}{\partial S^{2}}-r S \frac{\partial V}{\partial S},
$$

where $S(t)$ denotes the underlying asset, $t \in(0, T), T$ denotes the expiry date, $\sigma$ is the volatility (measures the standard deviation of the returns), and $r$ is the riskless interest rate.

In (29), the parameter $\sigma$ is constant since the transaction cost is taken as zero. Obviously, the $\sigma$ is not really a constant, and then we can obtain the nonlinear Black-Scholes equation as follows:

$$
\frac{\partial V}{\partial t}=r V-\frac{1}{2} \widetilde{\sigma}^{2}\left(t, S, \frac{\partial V}{\partial S}, \frac{\partial^{2} V}{\partial S^{2}}\right) S^{2} \frac{\partial^{2} V}{\partial S^{2}}-r S \frac{\partial V}{\partial S},
$$

where $\widetilde{\sigma}$ denotes a nonconstant volatility.

In order to solve the problem, it is necessary to perform a variable transformation as follows:

$$
x=\ln \left(\frac{S}{K}\right), \quad \tau=\frac{1}{2} \sigma^{2}(T-t), \quad u(x, \tau)=e^{-x} \frac{V(s, t)}{K} .
$$

Substituting (31) into (30), the following equation can be obtained:

$$
\frac{\partial u}{\partial t}=\frac{\widetilde{\sigma}^{2}}{\sigma^{2}}\left(\frac{\partial^{2} u}{\partial x^{2}}+\frac{\partial u}{\partial x}\right)+D \frac{\partial u}{\partial x}
$$

where

$$
D=\frac{2 r}{\sigma^{2}}, \quad x \in R, 0 \leq \tau \leq \widetilde{T}=\frac{\sigma^{2}}{2} .
$$

Initial condition

$$
u(x, 0)=\left(1-e^{-x}\right)^{+} \quad \text { for } x \in R .
$$

Boundary condition

$$
\begin{gathered}
u(x, \tau)=0 \quad \text { as } x \longrightarrow-\infty, \\
u(x, \tau) \sim 1-e^{-D \tau-x} \text { as } x \longrightarrow \infty .
\end{gathered}
$$

The initial condition is shown in Figure 1. According to the transformation relation (31), it is easy to understand that the point $x=0$ is corresponding to the strike price $S=$ $K$. Obviously, the initial solution curve is smooth in most positions except that near $x=0$, where a sharp steep wave happened. So, an adaptive numerical method is necessary to this problem.

The evolution of the call option price with the development of the parameter $t$ is illustrated in Figure 2, which shows that the volatility around the strike is greater and there is a sharp shock around it in the transformation form of the option price. The adaptive WPIM and VIM can capture it precisely; that is, there are more collocation points around this place than other places. This is helpful to improve the accuracy and efficiency.

In following, an adaptive interpolation wavelet numerical method is used to solve the nonlinear partial differential equation.

It is well known that the analytical solution of the linear Black-Scholes model for call option price $(C)$ can be obtained as follows:

$$
C=S \cdot N\left(d_{1}\right)-K e^{-r T} N(d 2),
$$

where

$$
d_{1}=\frac{\ln (S / K)+\left(r+(1 / 2) \sigma^{2}\right) T}{\sigma \sqrt{T}}, \quad d_{2}=d_{1}-\sigma \sqrt{T},
$$

where $C$ is the call price, $S$ is the underlying asset price, $K$ is the strike price, $r$ is the riskless rate, $T$ is the maturity, $\sigma$ is the volatility, and $N\left(d_{1}\right)$ expresses the normal distribution.

The error of the call option price between linear and nonlinear Black-Scholes models is shown in Figure 3. It is obvious that the error arising around the strike price, which expresses the nonlinear B-S model, and the coupling technique are effective. With the call option price that is going far away from the strike price, the error is becoming smaller and smaller, which shows that coupling technique is accurate and efficient. 


\section{Conclusion}

The coupling technique of VIM and WPIM developed in this paper can solve nonlinear partial differential equations successfully. Comparison between the numerical results of the linear and nonlinear Black-Scholes models illustrates that the proposed method is an accurate and efficient method for the nonlinear PDEs. In addition, as the coupling technique of VIM and WPIM for matrix differential equations has the uniform analytical solution, it can be easily used to solve various nonlinear problems.

\section{Acknowledgments}

The author would like to express his warmest gratitude to Professor Shuli Mei, for his instructive suggestions and valuable comments on the writing of this thesis. Without his invaluable help and generous encouragement, the present thesis would not have been accomplished. At the same time, the author is also grateful to the support of the National Natural Science Foundation of China (no. 41171337), the National Key Technology R and D Program of China (no. 2012BAD35B02), and the Project for Improving Scientific Research Level of Beijing Municipal Commission of Education.

\section{References}

[1] J. Wang, J. R. Liang, L. J. Lv, W. Y. Qiu, and F. Y. Ren, "Continuous time Black-Scholes equation with transaction costs in subdiffusive fractional Brownian motion regime," Physica A, vol. 391, no. 3, pp. 750-759, 2012.

[2] J. P. N. Bishwal, "Stochastic moment problem and hedging of generalized Black-Scholes options," Applied Numerical Mathematics, vol. 61, no. 12, pp. 1271-1280, 2011.

[3] T. K. Jana and P. Roy, "Pseudo Hermitian formulation of the quantum Black-Scholes Hamiltonian," Physica A, vol. 391, no. 8, pp. 2636-2640, 2012.

[4] M. K. Kadalbajoo, L. P. Tripathi, and A. Kumar, "A cubic Bspline collocation method for a numerical solution of the generalized Black-Scholes equation," Mathematical and Computer Modelling, vol. 55, no. 3-4, pp. 1483-1505, 2012.

[5] J. H. He, "Variational iteration method: a kind of nonlinear analytical technique: some exomples," International Journal of Non-Linear Mechanics, vol. 34, no. 4, pp. 699-708, 1999.

[6] J. H. He, "Variational iteration method for autonomous ordinary differential systems," Applied Mathematics and Computation, vol. 114, no. 2-3, pp. 115-123, 2000.

[7] J. H. He, X. H. Wu, and F. Austin, "The variational iteration method which should be followed," Nonlinear Science Letters A, vol. 1, pp. 1-30, 2007.

[8] J. H. He and X. H. Wu, "Variational iteration method: new development and applications," Computers \& Mathematics with Applications, vol. 54, no. 7-8, pp. 881-894, 2007.

[9] J. H. He, "Asymptotic methods for solitary solutions and compactons," Abstract and Applied Analysis, vol. 2012, Article ID 916793, 130 pages, 2012.

[10] G. C. Wu, "New trends in the variational iteration method," Communications in Fractional Calculus, vol. 2, no. 2, pp. 59-75, 2011.
[11] M. S. Tauseef, Y. Ahmet, A. S. Sefa, and U. Muhammad, "Modified variational iteration method for free-convective boundarylayer equation using padé approximation," Mathematical Problems in Engineering, vol. 2010, Article ID 318298, 11 pages, 2010.

[12] G. C. Wu, "Variational iteration method for $q$-difference equations of second order," Journal of Applied Mathematics, vol. 2012, Article ID 102850, 5 pages, 2012.

[13] H. Kong and L. L. Huang, "Lagrange multipliers of $q$-difference equations of third order," Communications in Fractional Calculus, vol. 3, no. 1, pp. 30-33, 2012.

[14] G. C. Wu and D. Baleanu, "Variational iteration method for the Burgers' flow with fractional derivatives-new Lagrange multipliers," Applied Mathematical Modelling, 2012.

[15] G. C. Wu, "Challenge in the variational iteration method-a new approach to the Lagrange multipliers," Journal of King Saud University-Science, 2012.

[16] S. L. Mei, Q. S. Lu, S. W. Zhang, and L. Jin, "Adaptive interval wavelet precise integration method for partial differential equations," Applied Mathematics and Mechanics, vol. 26, no. 3, pp. 364-371, 2005.

[17] S. L. Mei, C. J. Du, and S. W. Zhang, "Asymptotic numerical method for multi-degree-of-freedom nonlinear dynamic systems," Chaos, Solitons and Fractals, vol. 35, no. 3, pp. 536-542, 2008.

[18] S. L. Mei and S. W. Zhang, "Coupling technique of variational iteration and homotopy perturbation methods for nonlinear matrix differential equations," Computers \& Mathematics with Applications, vol. 54, no. 7-8, pp. 1092-1100, 2007.

[19] S. L. Mei, C. J. Du, and S. W. Zhang, "Linearized perturbation method for stochastic analysis of a rill erosion model," Applied Mathematics and Computation, vol. 200, no. 1, pp. 289-296, 2008.

[20] S. L. Mei, S. W. Zhang, and T. W. Lei, "On wavelet precise timeintegration method for Burgers equation," Chinese Journal of Computational Mechanics, vol. 20, no. 1, pp. 49-52, 2003. 


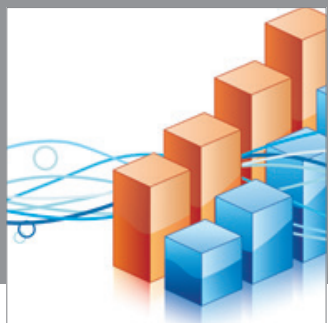

Advances in

Operations Research

mansans

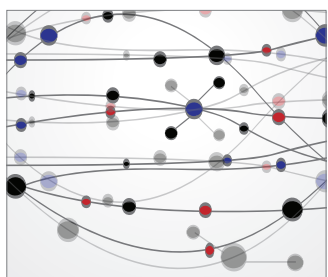

The Scientific World Journal
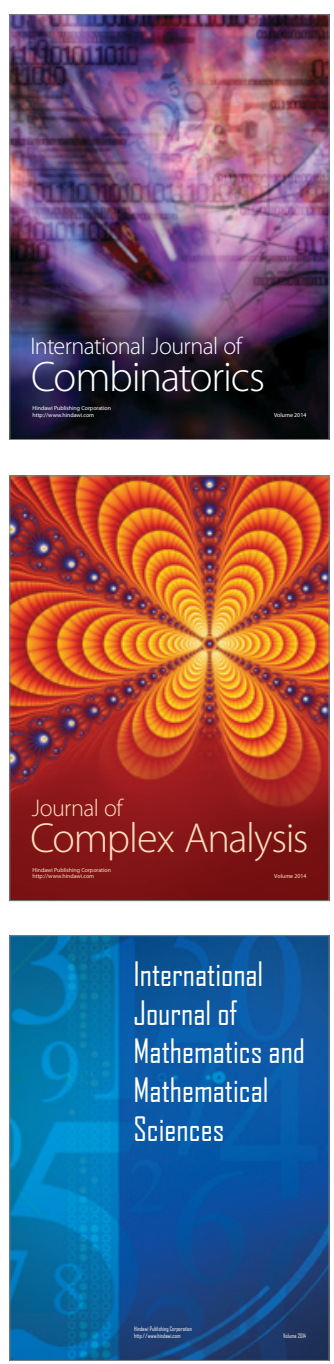
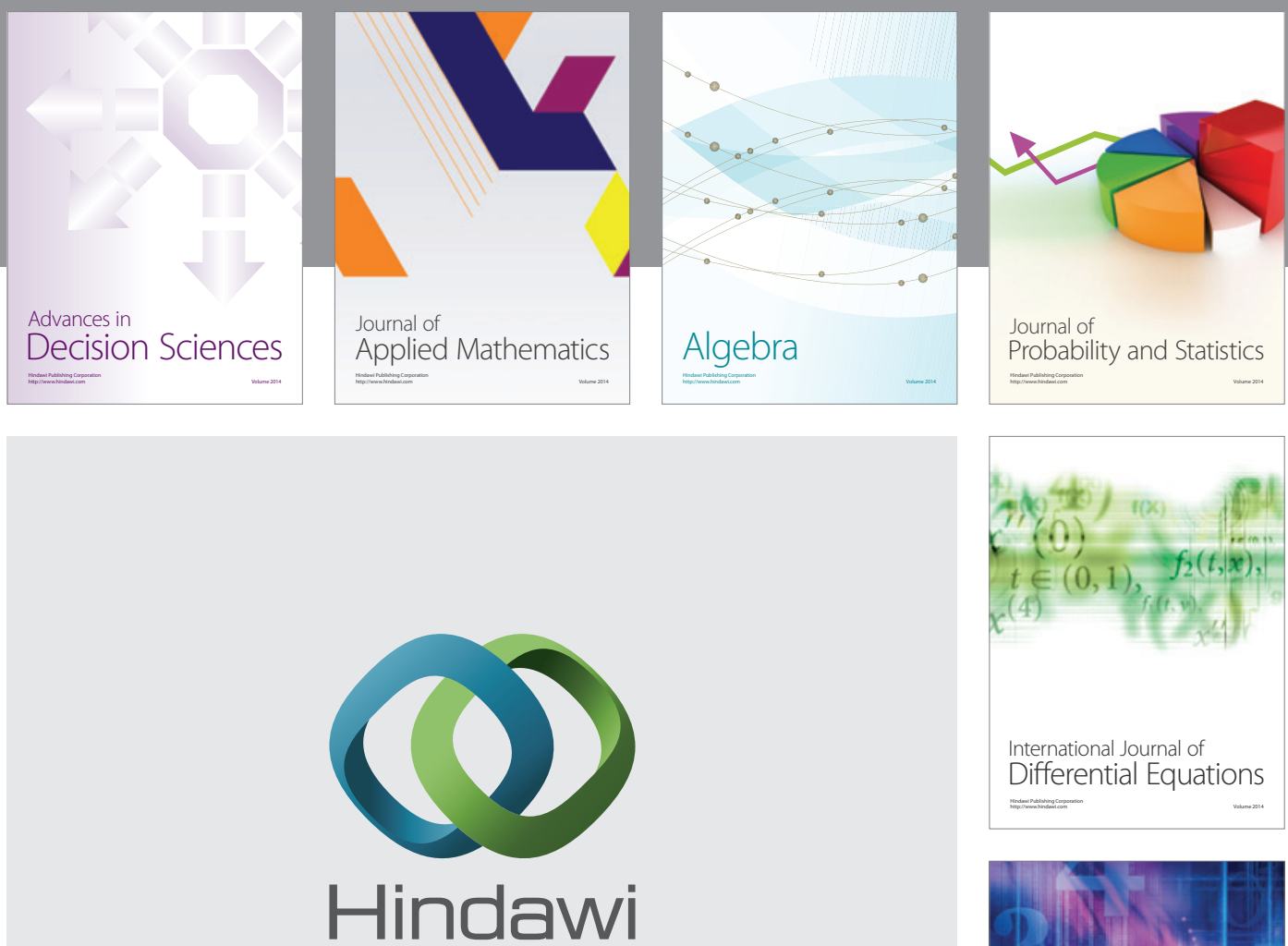

Submit your manuscripts at http://www.hindawi.com
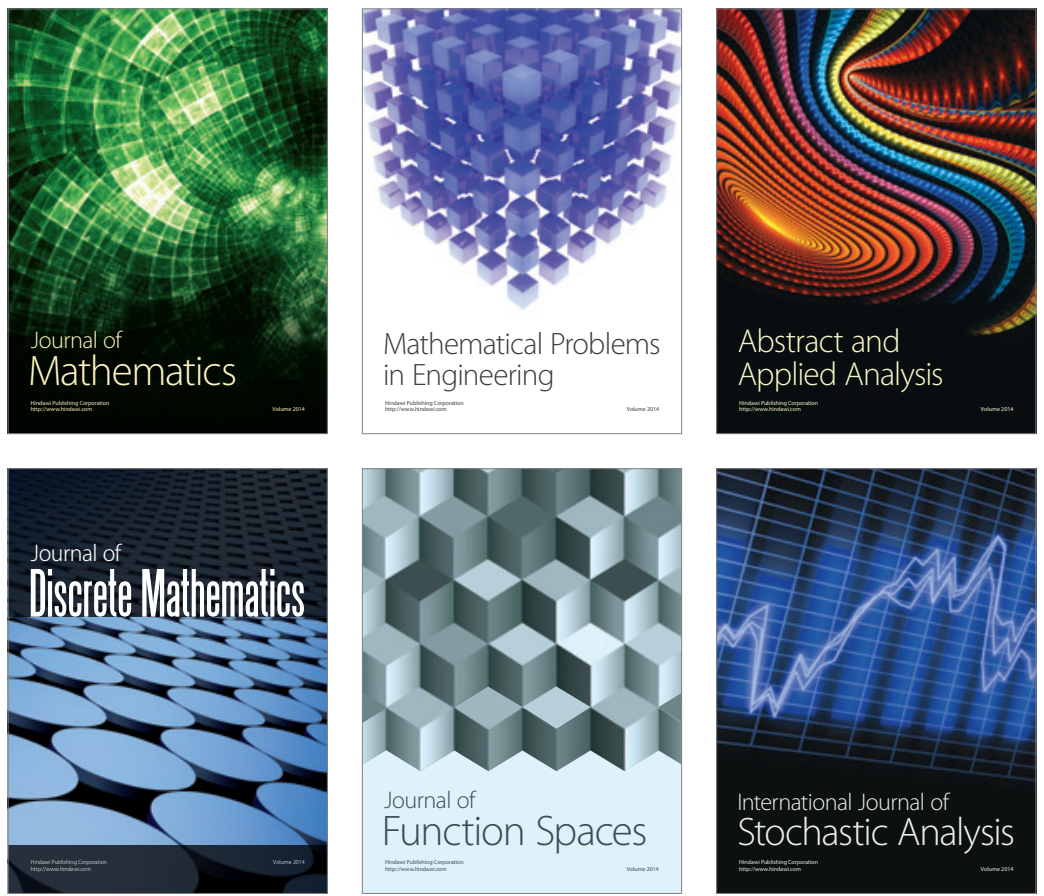

Journal of

Function Spaces

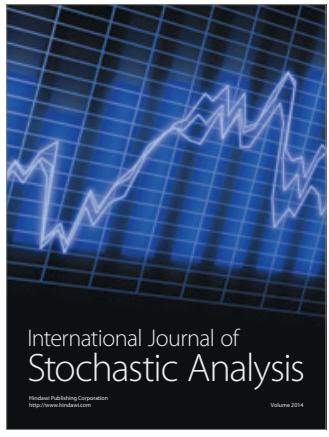

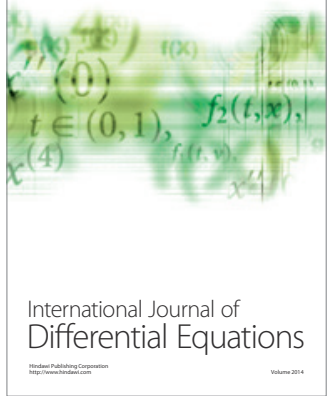
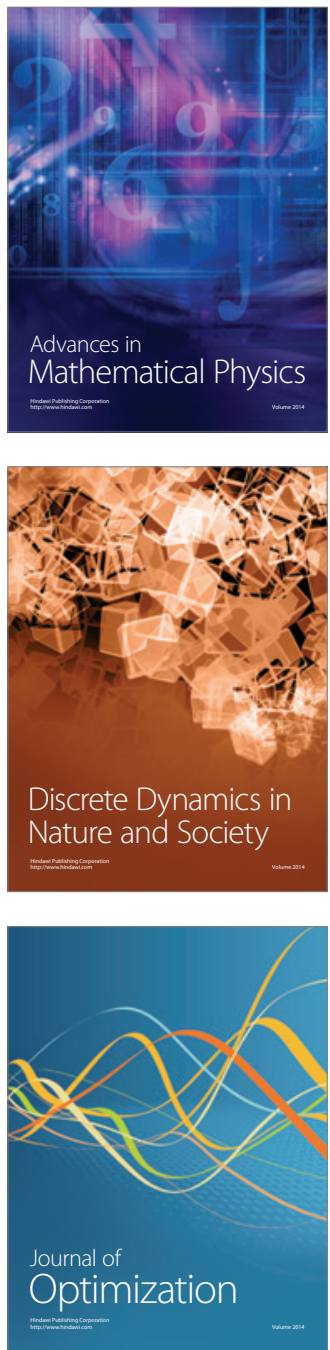